\title{
ON COISOTROPIC IMBEDDINGS OF PRESYMPLECTIC MANIFOLDS
}

\author{
MARK J. GOTAY
}

ABSTRACr. Existence and uniqueness theorems are proved for coisotropic imbeddings of presymplectic manifolds into symplectic manifolds.

I. Introduction. A manifold $M$ is presymplectic if it carries a distinguished closed 2-form $\omega$ of constant rank; if $\omega$ is nondegenerate, then $(M, \omega)$ is symplectic. Let $(M, \omega)$ be a presymplectic manifold. A coisotropic imbedding of $(M, \omega)$ into a symplectic manifold $(P, \Omega)$ is a closed imbedding $j: M \rightarrow P$ such that

(i) $j^{*} \Omega=\omega$,

(ii) $T M^{\perp} \subseteq T j(T M)$.

Such imbeddings play an important role in both the theory of constraints and quantization theory [1], [2], where $(M, \omega)$ represents the (degenerate) phase space of a physical system.

We show that every presymplectic manifold may be coisotropically imbedded in a symplectic manifold. Specifically, we prove the following

EXISTENCE THEOREM. There exists a symplectic structure on a neighborhood of the zero-section of $E^{*}$, where $E$ denotes the characteristic bundle of $(M, \omega)$. Moreover, $(M, \omega)$ may be coisotropically imbedded in this neighborhood as the zero-section.

Once existence has been established, we classify symplectic neighborhoods of coisotropic imbeddings. To this end it is useful to introduce, following Weinstein [3]-[5], the notion of a neighborhood equivalence of two imbeddings

$$
j_{1}: M \rightarrow\left(P_{1}, \Omega_{1}\right) \text { and } j_{2}: M \rightarrow\left(P_{2}, \Omega_{2}\right) \text {. }
$$

This consists of

(i) open neighborhoods $U_{i}$ of $j_{i}(M)$ in $P_{i}$,

(ii) a symplectomorphism $\psi:\left(U_{1}, \Omega_{1}\right) \rightarrow\left(U_{2}, \Omega_{2}\right)$ such that $\psi \circ j_{1}=j_{2}$.

We then prove a

LOCAL UNIQUENESS TheOREM. All coisotropic imbeddings of $(M, \omega)$ are neighborhood equivalent.

Thus, up to local symplectomorphism about $M$, there is a unique extension of $(M, \omega)$ to a symplectic manifold in which $M$ is coisotropic.

Received by the editors October 3, 1980.

1980 Mathematics Subject Classification. Primary 53C15; Secondary 57R40.

Key words and phrases. Symplectic, coisotropic imbeddings.

(c) 1982 American Mathematical Society 0002-9939/82/0000-0026/\$02.00 
The Local Uniqueness Theorem combined with the Existence Theorem shows that there exists a symplectic structure on a neighborhood of the zero-section of $E^{*}$ such that every symplectic manifold containing $(M, \omega)$ as a coisotropic submanifold is, near $M$, symplectomorphic to this neighborhood. The theorems in this article thus provide a complete local characterization of coisotropic imbeddings of presymplectic manifolds into symplectic manifolds.

These results are complementary to those obtained by Weinstein [3], [4] regarding isotropic imbeddings. In particular, the isotropic and coisotropic cases coincide when $\omega=0$, in which case $M$ is to be imbedded as a Lagrangian submanifold. Since in this instance $E^{*}=T^{*} M$, the two theorems imply Weinstein's result [3], [5] that every symplectic manifold containing $M$ as a Lagrangian submanifold is, near $M$, symplectomorphic to a neighborhood of the zero-section in $T^{*} M$.

Both of the theorems rely heavily upon Weinstein's extended version of the Darboux theorem [3], [5] which for convenience I state here.

EXTENSION TheOREM. Let $P$ be a manifold, $M \subseteq P$ a closed submanifold.

(A) Suppose that $\left(T_{M} P, \Omega\right)$ is a symplectic vector bundle and that $\Omega \mid T M$ is closed. Then $\Omega$ extends to a symplectic structure on a neighborhood of $M$ in $P$.

(B) Let $\Omega_{1}$ and $\Omega_{2}$ be symplectic forms on $P_{1}$ and $P_{2}$ respectively and let $M$ be imbedded in both $P_{1}$ and $P_{2}$. Suppose that $\left(T_{M} P_{1}, \Omega_{1}\right)$ and $\left(T_{M} P_{2}, \Omega_{2}\right)$ are isomorphic as symplectic vector bundles. Then there are neighborhoods $U_{i}$ of $M$ in $P_{i}$ and $a$ symplectomorphism $\psi:\left(U_{1}, \Omega_{1}\right) \rightarrow\left(U_{2}, \Omega_{2}\right)$ such that $\psi \mid M=\mathrm{id}_{M}$.

Notation and terminology are explained in [3].

II. Proof of the Existence Theorem. Let $(M, \omega)$ be presymplectic, and consider the vector bundle $\pi: E \rightarrow M$ with fiber

$$
E_{m}=\left\{x \in T_{m} M \mid \omega(x)=0\right\} .
$$

$E$ is the characteristic bundle of $(M, \omega)$; denote by $E^{*}$ the dual bundle.

Imbed $M$ as the zero-section of $E^{*}$. The strategy is to make $T_{M} E^{*}$ into a symplectic vector bundle over $M$ and then apply (A) of the Extension Theorem.

The restricted tangent bundle $T_{M} E^{*}$ has the canonical decomposition

$$
T_{M} E^{*}=T M \oplus E^{*}
$$

and, since $E$ is a subbundle of $T M$, one may further split

$$
T M=G \oplus E .
$$

Denote by $p r$ the induced projection $T_{M} E^{*} \rightarrow E \oplus E^{*} F$ or $m \in M$, let $\omega_{E}(m)$ denote the canonical symplectic structure on $E_{m} \oplus E_{m}^{*}$,

$$
\omega_{E}(m)\left(e \oplus e^{*}, f \oplus f^{*}\right)=f^{*}(e)-e^{*}(f) .
$$

Set

$$
\Omega_{G}=\pi^{*} \omega+\omega_{E} \circ(p r \times p r) .
$$

$\Omega_{G}$ is clearly smooth, well defined and nondegenerate. 
Thus $\left(T_{M} E^{*}, \Omega_{G}\right)$ is a symplectic vector bundle. Since $\Omega_{G} \mid T M=\omega$ is closed, (A) of the Extension Theorem applies. Consequently, $\Omega_{G}$ extends to a symplectic form on a neighborhood of $M$ in $E^{*}$ which clearly pulls back to $\omega$ on $M$.

It remains to show that $M$ is a coisotropic submanifold of $E^{*}$, i.e., that $T M^{\perp} \subseteq T M$ in $T_{M} E^{*}$. But this follows immediately from (4). Thus the Existence Theorem is proved.

III. Proof of the Local Uniqueness Theorem. Let $(M, \omega)$ be coisotropically imbedded in $(P, \Omega)$, so that $E=T M^{\perp} \subseteq T M$. Consider the symplectic vector bundle $\left(T_{M} P, \Omega\right)$, where $\Omega$ is the restriction to $T_{M} P$ of the symplectic structure on $P$, and let $G$ be any complement (2) of $E$ in $T M$. Since $G$ is a symplectic subbundle of $\left(T_{M} P, \Omega\right)$ so is $G^{\perp}$. Hence one has the splitting

$$
T_{M} P=G \oplus G^{\perp} \text {. }
$$

Now $E \subseteq G^{\perp}$ and, as $E^{\perp} \cap G^{\perp}=(E \oplus G)^{\perp}=E$, it follows that $E$ is a Lagrangian subbundle of $G^{\perp}$. Consequently [3], there exists a symplectomorphism

$$
\left(G^{\perp}, \Omega \mid G^{\perp}\right) \approx\left(E \oplus E^{*}, \omega_{E}\right),
$$

where $\omega_{E}$ is given by (3). Combining this result with (5) one obtains, mimicking (1), a vector bundle isomorphism

$$
T_{M} P \approx G \oplus E \oplus E^{*}
$$

Let $\Omega_{G}$ be the pullback of $\Omega$ to $G \oplus E \oplus E^{*}$ by the isomorphism (6). The symplectic structure $\Omega_{G}$ satisfies, and is in fact completely characterized by, the following three properties:

(i) $\Omega_{G} \mid(G \oplus E)=\omega$.

(ii) $\Omega_{G} \mid\left(E \oplus E^{*}\right)=\omega_{E}$.

(iii) $G^{\perp}=E \oplus E^{*}$.

It follows that $\Omega_{G}$ is given explicitly by (4), where $\pi: G \oplus E \oplus E^{*} \rightarrow G \oplus E$ and pr: $G \oplus E \oplus E^{*} \rightarrow E \oplus E^{*}$ are the projections.

Consequently, the symplectic vector bundle $\left(G \oplus E \oplus E^{*}, \Omega_{G}\right)$ depends only upon $M, \omega$ and the decomposition (2) of $T M$ and thus is independent of the imbedding space $(P, \Omega)$. If $\left(P^{\prime}, \Omega^{\prime}\right)$ is another symplectic manifold in which $(M, \omega)$ is coisotropically imbedded, then, the prior constructions give rise to vector bundle symplectomorphisms

$$
\left(T_{M} P, \Omega\right) \approx\left(G \oplus E \oplus E^{*}, \Omega_{G}\right) \approx\left(T_{M} P^{\prime}, \Omega^{\prime}\right)
$$

for some fixed (but irrelevant) splitting (2). We therefore conclude from (B) of the Extension Theorem that the coisotropic imbeddings $(M, \omega) \rightarrow(P, \Omega)$ and $(M, \omega) \rightarrow$ $\left(P^{\prime}, \Omega^{\prime}\right)$ are neighborhood equivalent. Thus there is but one neighborhood equivalence class of coisotropic imbeddings of $(M, \omega)$.

ACKNowledgemenTs. I thank J. Śniatycki and J. Marsden for their invaluable assistance. In particular, the idea of using the dual of the characteristic bundle as the imbedding space was communicated to me by J. Sniatycki, while the statement of the Existence Theorem was first formulated by J. Marsden. I am also indebted to A. Weinstein for his interest and helpful comments, and to the referee for suggesting substantial improvements in the proofs. 


\section{REFERENCES}

1. M. J. Gotay, Geometric quantization (M. J. Gotay, Ed.), University of Calgary, 1981.

2. M. J. Gotay and J. Sniatycki, Quantization of presymplectic dynamical systems via coisotropic imbeddings, Comm. Math. Phys. (to appear).

3. A. Weinstein, Lectures on symplectic manifolds, CBMS Regional Conf. Ser. in Math., no. 29, Amer. Math. Soc., Providence, R. I., 1977.

4. _ Neighborhood classification of isotropic imbeddings, Univ. of California, Berkeley, 1979 (preprint).

5. Advances in Math. 6 (1971), 329.

Department of Mathematics and Statistics, University of Calgary, Calgary, Alberta, Canada T2N 1N4 